\title{
Kinetics of Liquid Phase Semiconductor Photoassisted Reactions: Supporting Observations for a Pseudo-Steady-State Model
}

\author{
Andrew Mills, ${ }^{*}, \dagger$ Jishun Wang, ${ }^{\dagger}$ and David F. Ollis ${ }^{\ddagger}$ \\ Department of Pure \& Applied Chemistry, University of Strathclyde, Glasgow, G1 1XL, United Kingdom, and \\ Chemical Engineering Department, North Carolina State University, Raleigh, North Carolina 27695-7905
}

Received: April 14, 2006; In Final Form: May 31, 2006

\begin{abstract}
The kinetics of liquid phase semiconductor photocatalytic and photoassisted reactions are an area of some debate, reignited recently by an article by Ollis ${ }^{1}$ in which he proposed a simple pseudo-steady-state model to interpret the Langmuir-Hinshelwood type kinetics, commonly observed in such systems. In the current article, support for this model, over other models, is provided by a reinterpretation of the results of a study, reported initially in $1999,{ }^{2}$ of the photoassisted mineralization of 4-chlorophenol, 4-CP, by titania films and dispersions as a function of incident light intensity, I. On the basis of this model, these results indicate that 4-CP is adsorbed more strongly on $\mathrm{P} 25 \mathrm{TiO}_{2}$ when it is in a dispersed, rather than a film form, due to a higher rate constant for adsorption, $k_{1}$. In addition, the kinetics of 4-CP removal appear to depend on $I^{\beta}$, where $\beta=1$ or 0.6 for when the $\mathrm{TiO}_{2}$ is in a film or a dispersed form, respectively. These findings are discussed both in terms of the pseudo-steady-state model and other popular kinetic models.
\end{abstract}

\section{Introduction}

Semiconductor photoassisted reactions, especially ones in which the catalytic nature of the semiconductor has been fully established, ${ }^{3}$ i.e., photocatalytic reactions, continue to attract attention from both industry and academia. Several notable photocatalytic commercial products have emerged including the following: water purification systems, self-cleaning glass, and superhydrophilic tiles. ${ }^{4,5}$ The current high level of research activity is reflected by the fact that over 915 papers were published in 2005 alone with "photocatalyst" or "photocatalysis" in their titles, list of key words, or abstract. Much of this work has focused on the photocatalytic or photoassisted mineralization of organic pollutants, i.e.

$$
\text { organic }+ \text { oxygen } \underset{\mathrm{SC}}{\stackrel{h v \geq E_{\mathrm{bg}}}{\longrightarrow}} \mathrm{H}_{2} \mathrm{O}+\mathrm{CO}_{2}+\text { mineral acids }
$$

where $E_{\mathrm{bg}}$ is the band gap of the semiconductor, invariably $\mathrm{TiO}_{2} \cdot{ }^{6}$ A great deal of the early work in this area centered on the use of dispersions of semiconductor particles, whereas nowadays there is increasing interest in semiconductor films produced by CVD or sol-gel methods. ${ }^{5,7}$ In much of this work the kinetics of reaction 1 gave a good fit to a LangmuirHinshelwood (LH) rate form, i.e.

$$
-r_{\mathrm{A}}=\frac{\mathrm{k}_{\mathrm{LH}} K_{\mathrm{ads}}^{\mathrm{app}}[\mathrm{A}]}{1+K_{\mathrm{ads}}^{\mathrm{app}}[\mathrm{A}]}
$$

where $-r_{\mathrm{A}}$ is the rate of removal of the reagent of interest, A (usually an organic pollutant), and $k_{\mathrm{LH}}$ and $K_{\text {ads }}^{\text {app }}$ are the apparent maximum rate of the photoreaction and the apparent Langmuir adsorption constant, respectively. ${ }^{8}$ It has been long

*Address correspondence to this author.

$\dagger$ University of Strathclyde. E-mail: a.mills@strath.ac.uk.

$\doteqdot$ North Carolina State University. E-mail: ollis@eos.ncsu.edu. recognized that $K_{\text {ads }}^{\text {app }}$ is not usually the dark Langmuir adsorption constant, $K_{\text {ads }}$, and typically $K_{\text {ads }}^{\text {app }} \gg K_{\text {ads. }}{ }^{6,8,9}$

An early rationale for all of the then known features of the $\mathrm{LH}$ rate form in semiconductor photoassisted reactions, based on four possible hydroxyl radical mechanisms as the slow step, was provided by Turchi and Ollis in $1990 .{ }^{8}$ The features accounted for included not only the observation that $K_{\text {ads }}^{\text {app }} \gg$ $K_{\text {ads }}$ but also that $k_{\mathrm{LH}}$ was a function of absorbed light intensity, $I^{\beta}$, where $\beta=1.0$ and $\beta=0.5$ at low and high absorbed light intensities, respectively.

A substantial reevaluation of the mechanism of semiconductor photoassisted reactions has had to take place in recent years, upon the observation that $K_{\mathrm{ads}}^{\mathrm{app}}$ is, like $k_{\mathrm{LH}}$, light intensity dependent and possibly inversely related to $k_{\mathrm{LH}}{ }^{6 \mathrm{c}, 10}$ A simple mechanism that is able to account for all of the above has been proposed recently ${ }^{1}$ and is as follows:

$$
\begin{aligned}
& \mathrm{A}(\text { liquid }) \underset{k_{-1}}{\stackrel{k_{1}}{\longrightarrow}} \mathrm{A}(\mathrm{ads}) \\
& \mathrm{A}(\mathrm{ads}) \stackrel{k_{\mathrm{LH}}}{\longrightarrow} \text { products }
\end{aligned}
$$

In this mechanism it is assumed that the key step 4 involves the reaction of the adsorbed reactant and a photogenerated surface hydroxyl radical. Since the latter will be a function of absorbed light intensity, which in turn will be proportional to the incident light intensity, $I$, it follows that $k_{\mathrm{LH}}$ will also be a function of $I$. As suggested by Turchi and Ollis, ${ }^{8}$ it appears likely that $k_{\mathrm{LH}}$ is related to $I$ via the following expression:

$$
k_{\mathrm{LH}}=\alpha I^{\beta}
$$

where $\alpha$ is a proportionality constant and $\beta$ is 1.0 or 0.5 , as described earlier. In the most recent model, ${ }^{1}$ it is also assumed that a pseudo-steady-state hypothesis applies to the surface 
coverage of the reactant, $\theta_{\mathrm{A}}$, so that:

$$
\theta_{\mathrm{A}}=\frac{K_{\mathrm{ads}}^{\mathrm{app}}[\mathrm{A}]}{1+K_{\mathrm{ads}}^{\mathrm{app}}[\mathrm{A}]}
$$

where

$$
K_{\mathrm{ads}}^{\mathrm{app}}=1 / K_{\mathrm{diss}}^{\mathrm{app}}=\frac{k_{1}}{k_{-1}+\alpha I^{\beta}}
$$

and $K_{\text {diss }}^{\text {app }}$ is the apparent dissociation constant.

Assuming that $-r_{\mathrm{A}}=k_{\mathrm{LH}} \theta_{\mathrm{A}}$, it follows:

$$
-r_{\mathrm{A}}=\frac{k_{\mathrm{LH}}[\mathrm{A}]}{K_{\mathrm{diss}}^{\mathrm{app}}+[\mathrm{A}]}
$$

where $k_{\mathrm{LH}}$ is given by eq 5 and $K_{\text {diss }}^{\text {app }}$ by eq 7 . This pseudosteady-state approach predicts that both $k_{\mathrm{LH}}$ and $K_{\mathrm{diss}}^{\mathrm{app}}$ depend on the intensity of absorbed light raised to the same exponent. ${ }^{1}$ In support of the above mechanism, Ollis cited the results of three papers published in 2000, which revealed an intensity dependence for $K_{\text {ads }}^{\text {app }}$ when the reactant, A, was either phenol, ${ }^{10 a}$ acetophenone, ${ }^{10 \mathrm{~b}}$ or methyl viologen. ${ }^{10 \mathrm{c}}$ However, two of the papers ${ }^{10 b, c}$ contained only three points or less and the third, ${ }^{10 a}$ an excellent study of the photomineralization of $\mathrm{PhOH}$ by $\mathrm{TiO}_{2}$, appeared to be a special case for the Ollis model, where $\alpha I \gg$ $k_{-1}$ and the reaction approximates to adsorption followed by reaction. Encouragingly, further, possibly more striking, support for the pseudo-steady-state approach can be gleaned from the results of a study of light intensity effects on the kinetics of photomineralization of 4-chlorophenol, 4-CP, reported by Mills and Wang in 1999, in which the semiconductor sensitizer, Degussa $\mathrm{P} 25 \mathrm{TiO}_{2}$, was used as either a thin film or an aqueous dispersion. $^{2}$ In the latter work the substantial body of results on the $4-\mathrm{CP} / \mathrm{TiO}_{2}$ system is not a special case and so can be used to test the Ollis model much more fully; this paper is a reanalyzis of those results, ${ }^{2}$ based on the pseudo-steady-state approach of Ollis. ${ }^{1}$

\section{The Kinetics of the Photomineralization of 4-CP, Sensitized by $\mathrm{TiO}_{2}$}

In the latter work, ${ }^{2}$ P25 titanium dioxide from Degussa was used as the photosensitizer either as a film, supported on the glass of the photoreactor, or as a dispersion $\left(0.5 \mathrm{~g} \mathrm{dm}^{-3}\right)$. The aqueous reaction solutions under test $\left(100 \mathrm{~cm}^{3} ; \mathrm{pH} 2 ;\left(\mathrm{HClO}_{4}\right)\right.$, containing different levels of 4-CP $(0.1-1 \mathrm{mM}))$, were magnetically stirred $(600 \mathrm{rpm})$ at least $15 \mathrm{~min}$ before, as well as throughout, any irradiation. The solutions were also continually purged with oxygen. Other work showed that the rate of reaction was independent of stirrer speed when any $\mathrm{TiO}_{2}$ film was used as the photosensitizer, eliminating the possibility that the observed kinetics were due to external mass transfer. The Thiele modulus for the system was evaluated as $0.043-0.028$, implying little or no internal mass transfer effects for this system. ${ }^{11}$ HPLC was used to determine the initial rate of disappearance of 4-CP, i.e., $-r$, which was calculated over the first $20 \%$ of the reaction. Further details of the $\mathrm{TiO}_{2}$ film preparation and characterization are given in the original paper, along with a description of the glass Dreschel bottle photoreactor and surrounding irradiation system, comprising twelve, 8-W blacklight blue, UVA lamps with an aluminum reflector. ${ }^{2}$

The suitability of using 4-CP as a test reagent might be questioned initially given that, unlike $\mathrm{PhOH}$, it can be photore-
TABLE 1: LH Parameters, $k_{\mathrm{LH}}$ and $K_{\mathrm{ads}}^{\mathrm{app}}$, for the Photoassisted Destruction of 4-CP Mediated by $\mathrm{TiO}_{2}$ Film or Dispersion $^{a}$

\begin{tabular}{cccccc}
\hline & \multicolumn{2}{c}{$\mathrm{TiO}_{2}$ film } & & \multicolumn{2}{c}{$\mathrm{TiO}_{2}$ dispersion } \\
\cline { 2 - 3 }$I_{\text {rel }}$ & $\left(\mu \mathrm{M} \mathrm{min}^{-1}\right)$ & $\left(10^{3} \mathrm{M}^{-1}\right)$ & & $\begin{array}{c}k_{\mathrm{LH}} \\
\left(\mu \mathrm{M} \mathrm{min}^{-1}\right)\end{array}$ & $\begin{array}{c}K_{\text {ads }}^{\text {app }} \\
\left(10^{3} \mathrm{M}^{-1}\right)\end{array}$ \\
\hline 1 & $26.1( \pm 6.2)$ & $1.70( \pm 0.41)$ & & $13.6( \pm 2.4)$ & $4.52( \pm 0.87)$ \\
0.713 & $19.2( \pm 2.2)$ & $1.93( \pm 0.23)$ & & $11.2( \pm 1.5)$ & $4.63( \pm 0.69)$ \\
0.616 & $16.5( \pm 4.3)$ & $1.89( \pm 0.51)$ & & $10.2( \pm 0.9)$ & $4.27( \pm 0.40)$ \\
0.388 & $10.6( \pm 2.1)$ & $2.75( \pm 0.57)$ & & $8.1( \pm 0.5)$ & $5.11( \pm 0.38)$ \\
0.269 & $6.3( \pm 1.3)$ & $3.32( \pm 0.74)$ & & $6.9( \pm 0.6)$ & $5.00( \pm 0.50)$ \\
0.143 & $2.9( \pm 0.3)$ & $6.23( \pm 0.69)$ & & $3.9( \pm 0.3)$ & $8.36( \pm 0.77)$ \\
0.084 & $2.5( \pm 0.1)$ & $5.09( \pm 0.25)$ & & $2.8( \pm 0.2)$ & $9.08( \pm 0.74)$
\end{tabular}

${ }^{a}$ Derived from studies of the initial rate of removal of 4-CP as a function of [4-CP] at different relative light intensities and use of eq 2 to extract values for $k_{\mathrm{LH}}$ and $K_{\mathrm{abs}}^{\mathrm{app}}$ at the different light intensities.

duced, as well as oxidized. ${ }^{12,13}$ However, the unambiguous intermediates associated with this process, such as $\mathrm{PhOH}$ and bicyclic compounds, are only observed at $\mathrm{pH}>7$, and then at very low levels, since the initial hydroxyl phenyl radical is most likely formed at higher $\mathrm{pH}$ values. ${ }^{13}$ In this work, at $\mathrm{pH} 2$, in $0.01 \mathrm{M} \mathrm{HClO}_{4}$ and $\mathrm{O}_{2}$-saturated solution, the major intermediates observed are 4-chlorocatechol (4-CC) and hydroquinone (HQ); both of which are produced promptly upon UV illumination, with the level of 4-CC usually 3 times that of HQ. ${ }^{14}$ The level of benzoquinone (BQ) generated is typically 17 times less than that of 4-CC, and although BQ can be produced via a reductive as well as oxidative mechanism, its generation appears to track the production of HQ, as expected if it were derived only via an oxidative pathway. ${ }^{14}$ Similar intermediates and relative quantities have been reported for the same photoassisted reaction by others, ${ }^{15,16}$ and this and the above results suggest that it is reasonable to assume that the photodegradation of 4-CP occurs predominantly via an oxidative pathway, as in the case of $\mathrm{PhOH}$, for example. ${ }^{12}$

Another possible concern is wavelength selectivity, since a blacklight blue lamp, which emits $365 \pm 17 \mathrm{~nm}$ light, is used here as the illumination source and others have noted some spectral selectivity over this wavelength range using the 4-CP/ $\mathrm{TiO}_{2}$ system. ${ }^{12}$ This does not appear to be an issue in this work since a separate experiment was performed in which the light from a BLB light, filtered with a $365 \pm 5 \mathrm{~nm}$ interference filter, was used as the light source irradiating a $4-\mathrm{CP} / \mathrm{TiO}_{2}$ system. The results showed the intermediate product distribution was the same as that observed by using the full output of the BLB: a product distribution dominated by 4-CC and indicative of a photooxidative mechanism. Stafford et al. using either a 450-W medium-pressure $\mathrm{Hg}$ lamp or a BLB in the irradiation of the 4- $\mathrm{CP} / \mathrm{TiO}_{2}$ system also noted that in both cases the major reaction intermediate was $4-C C .{ }^{17}$

The results of this work with both $\mathrm{TiO}_{2}$ films and dispersions revealed that the kinetics of photodestruction of 4-CP give a good fit to a $\mathrm{LH}$ rate form, over a wide range of different light intensities. These results allowed the extraction of values for $k_{\mathrm{LH}}$ and $K_{\mathrm{ads}}^{\mathrm{app}}$ for a $\mathrm{TiO}_{2}$ film and dispersion for different incident light intensities, the results of which are given in Table 1 and illustrated in Figures 1 and 2, respectively. An initial analysis $^{2}$ of these data revealed that $k_{\mathrm{LH}}$ and $K_{\text {ads }}^{\text {app }}$ are directly proportional to (a) $I^{1.0}$ and $I^{-0.5}$, respectively, when a $\mathrm{TiO}_{2}$ film is used as the photosensitizer, and (b) $I^{0.64}$ and $I^{-0.32}$, respectively, when a $\mathrm{TiO}_{2}$ dispersion is used instead. At the time of their production these results were novel, but also hard to explain, without invoking rather involved and possibly unlikely mechanisms. ${ }^{2}$ Even with the advent of other studies, supporting 

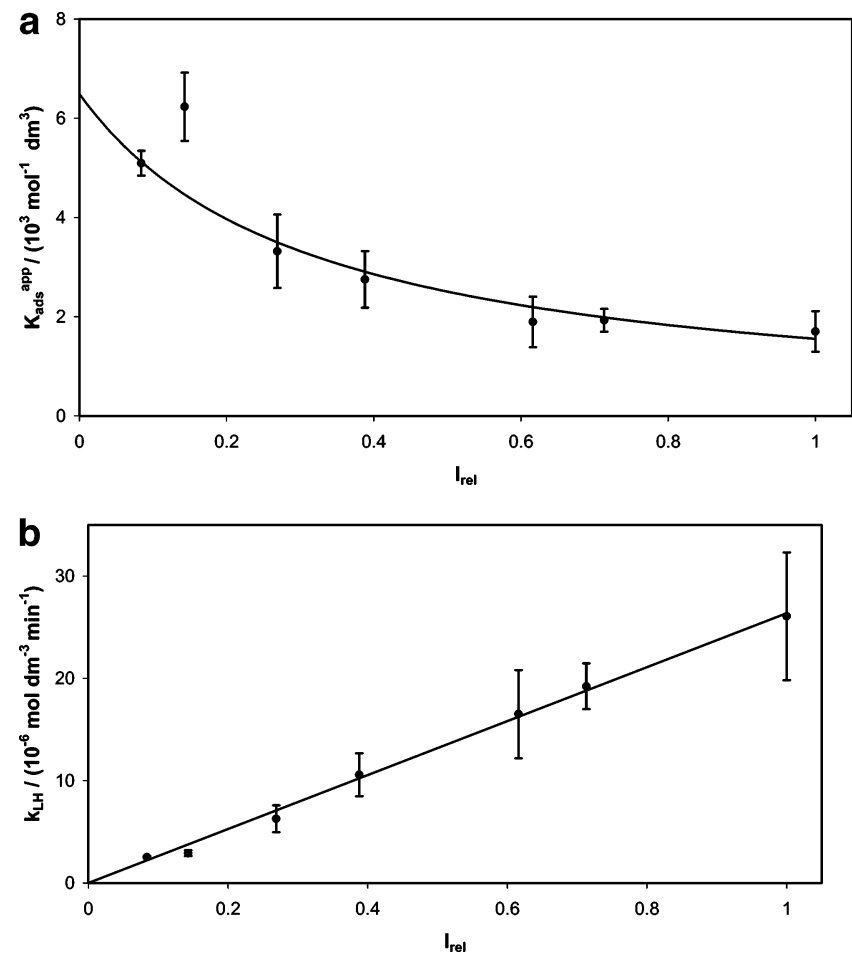

Figure 1. Variation in (a) $k_{\mathrm{LH}}$ and (b) $K_{\mathrm{abs}}^{\mathrm{app}}$, calculated from rate data using eq 2 , as a function of a relative incident light intensity, $I_{\text {rel }}\left(I_{\text {rel }}=\right.$ $1 \equiv 6.4 \times 10^{15}$ photons $\left.\mathrm{cm}^{-2} \mathrm{~s}^{-1}\right)$ for the initial photoassisted removal of 4-CP sensitized by a $\mathrm{TiO}_{2}$ film $\left(0.102 \mathrm{mg} \mathrm{cm}{ }^{-2}, 120.5 \mathrm{~cm}^{2}\right)$. In both $\mathrm{a}$ and $\mathrm{b}$ the solid lines have been calculated by using eqs 5,7 , and 8 of the pseudo-steady-state model and the optimized fit values, for $\alpha, k_{1}, k_{-1}$, and $\theta$, given in Table 2 .
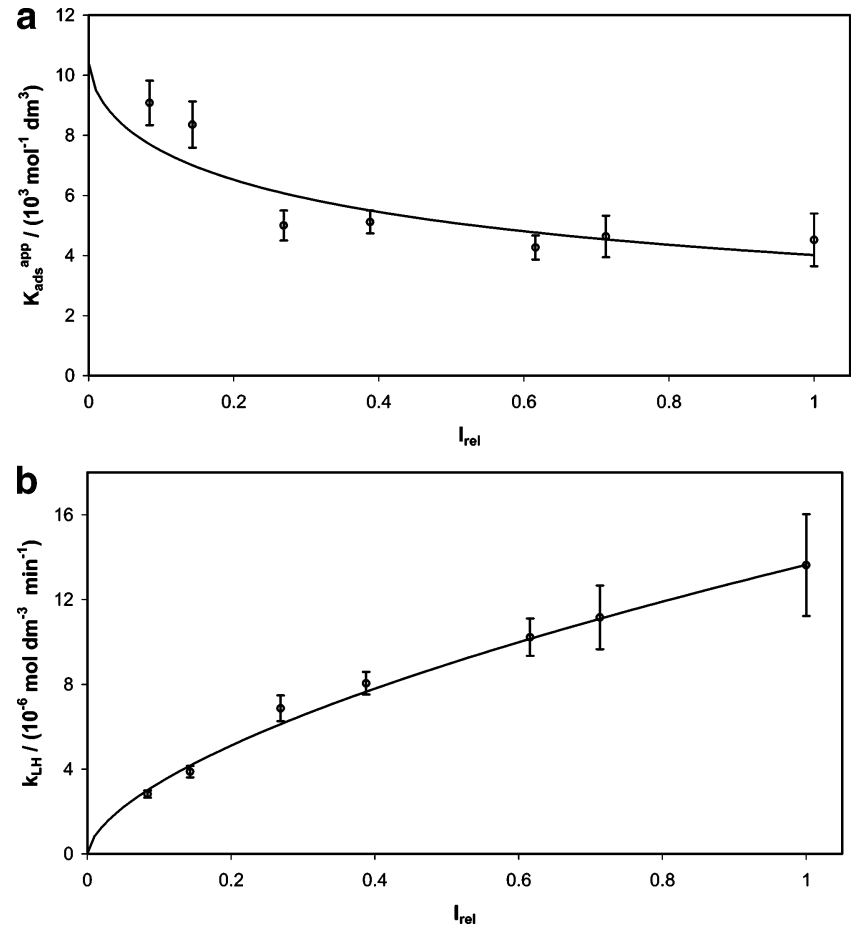

Figure 2. Variation in (a) $k_{\mathrm{LH}}$ and (b) $K_{\mathrm{abs}}^{\mathrm{app}}$ as a function of $I_{\mathrm{rel}}$ for the photoassisted removal of 4-CP sensitized by a $\mathrm{TiO}_{2}$ dispersion $(0.5 \mathrm{~g}$ $\mathrm{dm}^{-3} ; 100 \mathrm{~cm}^{3}$ ). In both a and $\mathrm{b}$ the solid lines have been calculated by using eqs 5, 7, and 8 and the optimized fit values, for $\alpha, k_{1}, k_{-1}$, and $\theta$, given in Table 2 .

the above novel discovery that $K_{\text {ads }}^{\text {app }}$ was intensity dependent, ${ }^{10}$ and the emergence of possible alternative mechanisms, ${ }^{10 a}$ these results resisted ready interpretation. For example, the revised
LH or Eley-Ridal (ER) based kinetic models of Emeline et al. ${ }^{10 a}$ provided an excellent rationale for the dependence they observed of $k_{\mathrm{LH}}$ and $K_{\mathrm{ads}}^{\mathrm{app}}$ on $I$ and $I^{-1}$, respectively, using phenol as the reactant and P25 dispersions as the photosensitizer. Encouragingly, the variation of $K_{\text {ads }}^{\text {app }}$ and $k_{\mathrm{LH}}$ with light intensity for the photoassisted removal of 4-CP observed by Mills and Wang, ${ }^{2}$ and reported in Table 1, can be fitted very well to the intensity LH model reported by Emeline et al. ${ }^{10 a}$ However, a key feature of both the revised LH and ER models is the prediction that 1/ $K_{\text {ads }}^{\text {app }}$ vs $k_{\mathrm{LH}}$ should be a straight line with zero intercept and, as we shall see, this does not fit well with the 4-CP data given in Table 1 and illustrated in Figures 1 and 2.

In a recent paper, ${ }^{1}$ Ollis showed that the pseudo-steady-state model gives a good fit to the data reported by Emeline et al. for the photoassisted removal of phenol ${ }^{10 a}$ by Degussa P25. It now appears that this same model also provides a reasonable fit to the more challenging data reported by Mills and Wang, ${ }^{2}$ on the photoassisted removal of 4-CP sensitized by Degussa P25, summarized in Table 1 and illustrated in Figures 1 and 2. Thus, as predicted by eq 7 for $\mathrm{TiO}_{2}$ films, a plot of the values for $K_{\text {diss }}^{\text {app }}\left(=1 / K_{\text {abs }}{ }^{\text {app }}\right)$ in Table 1 vs $I^{\beta}$, where $\beta=1.0$, yields a good straight line from which values for $k_{1} / k_{-1}$ and $\alpha / k_{1}$ can be obtained, based on the pseudo-steady-state model. A similar plot, using the results associated with the $\mathrm{TiO}_{2}$ dispersions in Table 1 , with $\beta=0.6$, also generates a good straight line. The values of $k_{1} / k_{-1}$ and $\alpha / k_{1}$ derived from these plots are collected in Table 2 and were used, in association with eqs 5, 7, and 8, to generate the solid line model fits to the data shown in Figures 1 and 2.

One of the predictions of the pseudo-steady-state model, ${ }^{1}$ in contrast to other models, ${ }^{10 \mathrm{a}}$ is that a plot of the data in the form $1 / K_{\text {abs }}{ }^{\text {app }}$ vs $k$ should be a straight line of gradient $=1 / k_{1}$ and intercept $=k_{-1} / k_{1}=K_{\text {diss }}\left(=1 / K_{\text {ads }}\right)$. The data for the $\mathrm{TiO}_{2}$ films and dispersions, given in Table 1 for the photooxidation of 4-CP, were plotted in this form and the results are illustrated in Figure 3 , parts a and b, respectively. From these results it would appear that the model, once again, provides a reasonable fit to the data. From the gradient and intercepts of the lines of best fit, values for $K_{\text {diss }}, K_{\text {ads }}, k_{1}$, and $k_{-1}$ were obtained for both the $\mathrm{TiO}_{2}$ films and dispersions and are collected in Table 2.

From the estimated values of $K_{\text {ads }}$ given in Table $2(6.3 \times$ $10^{3}$ (films) and $10.8 \times 10^{3} \mathrm{M}^{-1}$ (powder dispersions)) it is clear that they are much greater than that measured for the dark adsorption of 4-CP on $\mathrm{TiO}_{2}$ powder dispersions $\left(1.3 \times 10^{2}\right.$ $\left.\mathrm{M}^{-1}\right) .{ }^{14}$ This is not unusual, as noted earlier by Ollis, ${ }^{1}$ in reference to his examples, ${ }^{10 a, b}$ in his original paper and is due to the reaction sites associated with the process of photosensitization being different, e.g., a small subset of sites or only produced under illumination, to those probed by dark adsorption measurements.

From the values of $k_{1}$ and $k_{-1}$ in Table 2, it appears that 4-CP is adsorbed more strongly on $\mathrm{P} 25 \mathrm{TiO}_{2}$ when it is in dispersed, rather than film form, due to a higher rate constant for adsorption, $k_{1}$. These results suggest that the particle-glass wall interactions and increased particle-particle interactions associated with the films somehow adversely affect the kinetics of adsorption, rather than desorption. The greatest difference between the $\mathrm{TiO}_{2}$ films and dispersions, however, is the dependence upon the incident light intensity, with $\beta$ equal to 1.0 for films and 0.6 for dispersions. This difference may arise due to a higher threshold at which $\beta$ changes in value from 1.0 to 0.5 for films, where the particles are permanently intimately linked, compared to dispersions, where they are more isolated. Interestingly, Ahmed et al., using much higher intensities of $(2.0-0.7) \times 10^{17}$ photons $\mathrm{cm}^{-2} \mathrm{~s}^{-1}$, have also found that the 
TABLE 2: Optimized Fit Parameters to the 4-CP Kinetic Data, Based on the Pseudo-Steady-State Model

\begin{tabular}{lcccccr}
\hline & $\beta^{a}$ & $\alpha\left(\mu \mathrm{M} \mathrm{min}^{-1}\right)^{b}$ & $k_{-1} / k_{1}(\mu \mathrm{M})^{c}$ & $k_{1}\left(\min ^{-1}\right)^{c}$ & $k_{-1}\left(\mu \mathrm{M} \mathrm{min}^{-1}\right)^{c}$ & $K_{\text {ads }}\left(10^{3} \mathrm{M}^{-1}\right)^{c}$ \\
\hline $\mathrm{TiO}_{2}$ film & $1.02( \pm 0.06)$ & $26.4( \pm 0.7)$ & $160( \pm 30)$ & $0.054( \pm 0.006)$ & $8.6( \pm 1.9)$ & $6.3( \pm 1.2)$ \\
$\mathrm{TiO}_{2}$ dispersion & $0.60( \pm 0.03)$ & $13.8( \pm 0.6)$ & $92( \pm 22)$ & $0.087( \pm 0.019)$ & $8.0( \pm 2.6)$ & $10.8( \pm 2.6)$
\end{tabular}

${ }^{a}$ Derived from plots of $\log k_{\mathrm{LH}}$ vs $I_{\text {rel }}{ }^{b}$ Derived from plots of $k_{\mathrm{LH}}$ vs $I_{\mathrm{rel}}{ }^{\theta} .{ }^{c}$ Derived from plots of $1 / K_{\mathrm{ads}}^{\mathrm{app}}$ vs $k_{\mathrm{LH}}$.
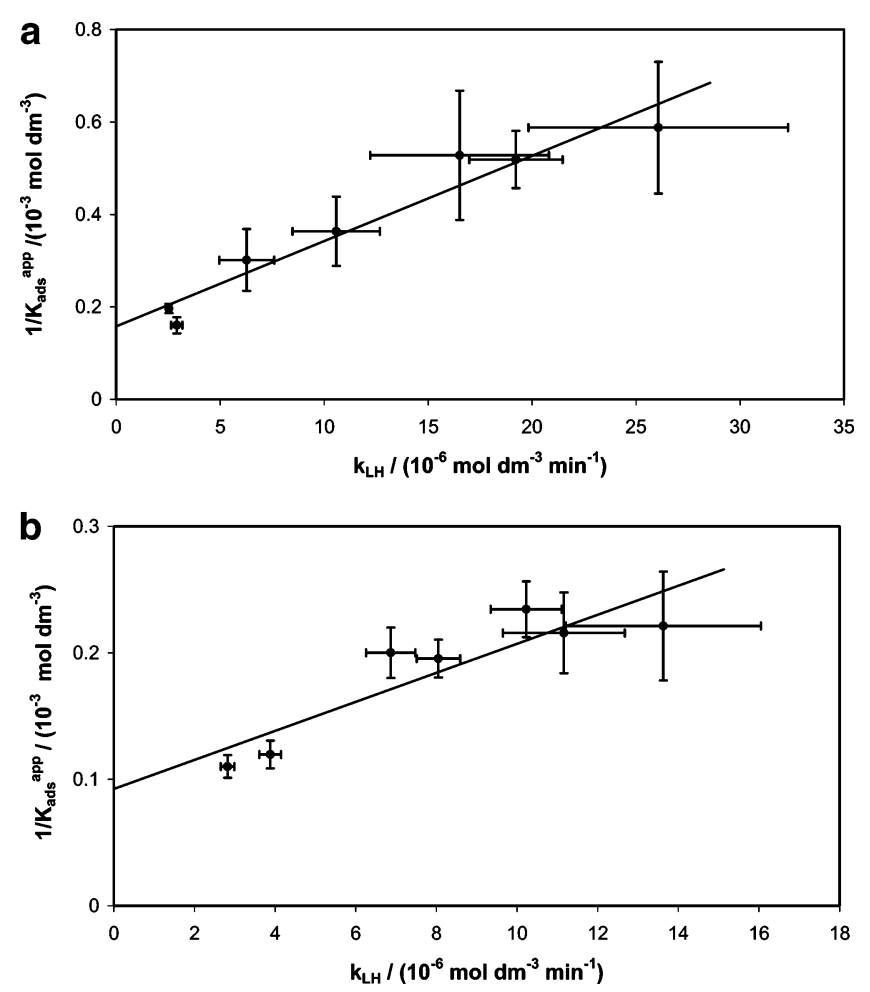

Figure 3. Plot of $1 / K_{\mathrm{abs}}^{\mathrm{app}}$ vs $k_{\mathrm{LH}}$ calculated by using eq 2 for reaction 1, using 4-CP as the organic, sensitized by (a) a $\mathrm{TiO}_{2}$ film and (b) a $\mathrm{TiO}_{2}$ dispersion. In both $\mathrm{a}$ and $\mathrm{b}$ the solid lines are the lines of best fit based on eq 7 and the optimized fit values, for $\alpha, k_{1}, k_{-1}$, and $\theta$, given in Table 2.

apparent rate constant for the removal of $4-\mathrm{CP}$ by a $\mathrm{P}_{25 \mathrm{TiO}_{2}}$ film is proportional to intensity. ${ }^{18}$

In contrast to films, the photoassisted destruction of 4-CP, sensitized by $\mathrm{TiO}_{2}$ dispersions, is usually reported as having a sublinear dependence upon intensity. For example, Pichat et al. ${ }^{19}$ have reported a value of 0.5 for $\beta$, using the high-intensity range $(3-90) \times 10^{15}$ photons $\mathrm{cm}^{-2} \mathrm{~s}^{-1}$ and Stafford et al. ${ }^{17}$ have reported a value of 0.8 . Egerton and King reported ${ }^{20}$ the transition of $\beta$ from a value of 1 to 0.5 occurs around an incident light intensity of $6 \times 10^{14}$ photons $\mathrm{cm}^{-2} \mathrm{~s}^{-1}$. In our work, the incident light intensities used spanned the range $(0.5-6) \times 10^{15}$ photons $\mathrm{cm}^{-2} \mathrm{~s}^{-1}$ and so it was perhaps not too surprising to calculate a value of $\beta=0.6$ for the photodestruction of 4-CP by $\mathrm{TiO}_{2}$ dispersions. Interestingly, in the kinetic study of reaction 1, using phenol as the organic, carried out by Emeline et al., ${ }^{10 a}$ a much higher range of incident intensities, $(6.6-110) \times 10^{15}$ photons $\mathrm{cm}^{-2} \mathrm{~s}^{-1}$ at $365 \mathrm{~nm}$, were employed, and so, given eq 7 , it is not too surprising that $\alpha I \gg k_{-1}$, the value of which appears negligibly small, and that, under such conditions, the reaction approximates to adsorption followed by reaction, i.e., two irreversible steps in series:

$$
\mathrm{A} \stackrel{k_{1}}{\longrightarrow} \mathrm{A}_{\mathrm{ads}} \stackrel{k_{\mathrm{LH}}}{\longrightarrow}
$$

as noted by Ollis. ${ }^{1}$ However, it is somewhat surprising to note that in this same work $^{8 \mathrm{a}}$ the value of $\beta$ appears to have a value of unity rather than 0.5 . Indeed, it has been argued recently by the same authors ${ }^{21}$ that in most photocatalytic work the free photogenerated carrier concentration in $\mathrm{TiO}_{2}$ will scale almost linearly at absorbed light intensities $<\sim 10^{16}$ photons $\mathrm{cm}^{-2} \mathrm{~s}^{-1}$ and so $\beta$ will only exhibit a value of unity. The results reported here, in which $k_{\text {obs }}$ is found to be directly related to $I^{0.6}$, for the 4- $\mathrm{CP} / \mathrm{TiO}_{2}$ system appears at odds with this assertion and although the value of $\beta=0.6$ can be interpreted by using the intensity-dependent LH model of Emeline et al., ${ }^{10 a, 21}$ the latter model fails in other important aspects as noted above. Indeed, a great many more detailed kinetic studies of reaction 1 and its dependence upon the concentration of the organic under test and incident light intensity will have to be carried out in both solution and the gas phase before a good broad understanding of the underlying reaction mechanism(s) can be claimed with confidence.

\section{Conclusions}

The Langmuir-Hinshelwood type kinetics of the photoassisted mineralization of 4-chlorophenol, 4-CP, by titania films and dispersions as a function of incident light intensity, $I$, reported many years ago, ${ }^{2}$ can be readily interpreted by using the simple pseudo-steady-state model reported by Ollis recently. ${ }^{1}$ On the basis of this model, the results show that 4-CP is adsorbed more strongly on $\mathrm{P} 25 \mathrm{TiO}_{2}$ when it is in a dispersed, rather than a film form, due to a higher rate constant for adsorption, $k_{1}$, and that the kinetics of 4-CP removal appear to depend on $I^{\beta}$, where $\beta=1$ or 0.6 when the $\mathrm{TiO}_{2}$ is in a film or dispersed form, respectively. These findings fit very well with the pseudo-steady-state model but not the kinetic models, and some of their underlying assumptions, reported by Emeline et al. ${ }^{10 a, 21}$ However, as noted earlier, it could be argued that 4-CP is a poor test regent, which exhibits kinetics complicated by reductive as well as oxidative pathways and wavelength selectivity. It could also be that the apparent different power dependencies of the initial rate upon $I$ for films and powders reported here are somehow the result of differences in light distribution, rather than an illustration of surface and recombination control. Thus, this study, with its not insignificant error bars, is not without its concerns and the apparent conflict in kinetic models will require further work before it is fully resolved.

\section{References and Notes}

(1) Ollis, D. F. J. Phys Chem. B 2005, 109, 2439.

(2) Mills, A.; Wang, J. Z. Phys. Chem. 1999, 2132, 49.

(3) Serpone, N.; Emerline, A. V. Int. J. Photoenergy 2002, 4, 91.

(4) Mills, A.; Lee, S. K. J. Photochem. Photobiol., A 2002, 152, 233.

(5) Fujishiam, A.; Hashimoto, K.; Watanabe, T. $\mathrm{TiO}_{2}$ Photocatalysis: Fundamentals and Applications; BKC Inc.: Tokyo, Japan, 1999.

(6) (a) Mills, A.; LeHunte, S. J. Photochem. Photobiol., A 1997, 108, 1. (b) Ollis, D. F.; Pelizetti, E.; Serpone, N. In Photocatlysis: Fundamentals and Applications; Serpone, N., Pelizetti, E. Eds.; Wiley-Interscience: New York, 1989. (c) Hoffmann, M. R.; Martin, S. T.; Choi, W.; Bahnemann, D. W. Chem. Rev. 1995, 95, 69. (d) Fox, M. A.; Dulay, M. T. Chem. Rev. 1993, 93, 34.

(7) (a) Mills, A.; Hill, G.; Bhopal, S.; Parkin, I. P.; O’Neill, S. A. J. Photochem. Photobiol., A 2003, 160, 185. (b) O'Neill, S. A.; Clark, R. H.; Parkin, I. P.; Elliott, N.; Mills, A. Chem. Mater. 2003, 15, 46.

(8) Turchi, C.; Ollis, D. F. J. Catal. 1990, 122, 178. 
(9) Cunningham, J.; Sedlak, P. J. Photochem. Photobiol., A 1994, 77,

(10) (a) Emeline, A. D.; Ryabchuk, V. K.; Serpone, N. J. Photochem. Photobiol., A 2000, 133, 89. (b) Xu, Y.; Langford, C. H. J. Photochem. Photobiol., A 2000, 133, 67. (c) Martyanov, I.; Savinov, E. J. Photochem. Photobiol., A 2000, 134, 219.

(11) Levenspiel, O. Chemical Reaction Engineering, 3rd ed.; Wiley: New York, 1999.

(12) Emerline, A.; Salinaro, A.; Serpone, A. J. Phys. Chem. B 2000, 104,11202

(13) Theurich, J.; Lindner, M.; Bahnemann, D. W. Langmuir 1996, 12, 6368.

(14) Mills, A.; Morris, S.; Davies, R. J. Photochem. Photobiol., A 1993, $70,183$.
(15) Vinodgopal, K.; Stafford, U.; Gray K. A.; Kamat, P. V. J. Phys. Chem. B 1994, 98, 6797.

(16) Stafford, U.; Gray, K. A.; Kamat, P. V. J. Phys. Chem. B 1994, $98,6343$.

(17) Stafford, U.; Gray, K. A.; Kamat, P. V. J. Catal. 1997, 167, 25.

(18) Ahmed, S.; Kemp, T. J.; Unwin, P. R. J. Photochem. Photobiol., A 2001, 141, 69 .

(19) Al-Fayyed, G.; D’Oliveira, J. C.; Pichat, P. J. Photochem. Photobiol., A 1991, 58, 99.

(20) Egerton, T. A.; King, C. J. J. Oil. Colour Chem. Assoc. 1979, 62, 386.

(21) Emeline, A. D.; Ryabchuk, V. K.; Serpone, N. J. Phys Chem. B $\mathbf{2 0 0 5}, 109,19515$. 